\title{
A multi-domain decomposition based Fourier finite element method for the simulation of 3D marine CSEM measurements
}

\author{
Shaaban A. Bakr • David Pardo
}

Received: date / Accepted: date

\begin{abstract}
We introduce a multi-domain decomposition Fourier finite element (MDDFFE) method for the simulation of three-dimensional (3D) marine controlled source electromagnetic (CSEM) measurements. The method combines a 2D finite element (FE) method in two spatial dimensions with a hybrid discretization based on a Fourier FE method along the third dimension. The method employs a secondary field formulation rather than the full field formulation. By using the secondary field formulation, we avoid to numerically model the source singularities and reduce the effect of the air layer. We apply the MDDFFE method to several synthetic marine CSEM examples exhibiting bathymetry and/or multiple 3D subdomains. Numerical results show that the use of the MDDFFE method reduces the problem size by as much as $87 \%$ in terms of the number of unknowns, without any sacrifice in accuracy.
\end{abstract}

Keywords Multi-domain decomposition · finite element method $\cdot$ secondary field formulation · Fourier series · marine CSEM.

\section{S. A. Bakr}

Basque Center for Applied Mathematics (BCAM), Mazarredo 14, E48009 Bilbao, Spain.

Department of Mathematics, Assiut University, Assiut 71516, Egypt.

E-mail: shaaban.bakr1@gmail.com

D. Pardo

University of the Basque Country (UPV/ EHU), Bilbao, Spain.

Basque Center for Applied Mathematics (BCAM), Bilbao, Spain.

Ikerbasque, Bilbao, Spain.

E-mail: dzubiaur@gmail.com

\section{Introduction}

A map of the Earth's subsurface is employed in multiple applications such as $\mathrm{CO}_{2}$ storage, hydrocarbon extraction, mining, and geothermal energy production, among others. To obtain such a map, multiple electromagnetic (EM), elasto-acoustic, and nuclear measurements can be recorded, both acquired from the surface or by employing borehole logging instruments. These measurements are subsequently inverted to obtain the material properties of the Earth's subsurface, and hence, to image it.

As an example of surface-based measurements, we consider a marine controlled source EM (CSEM) acquisition system [12]. It consists of a towed electric-dipole source and a number of seafloor electric and magnetic field receivers, as illustrated in Fig. 1. The towed source repeatedly excites electric fields at different locations, and the response after interacting with the geological formation is recorded by the receivers. Depending on the depth of the target area, the transmitter operating frequencies may range between 0.1 and $10 \mathrm{~Hz}$ and the source-receiver offsets can be up to $10 \mathrm{~km}$, see, e.g., [13]. Solving the forward problem then entails simulating the EM signals for all involved source positions and frequencies.

Utilization of marine CSEM data for identification of oil and gas reservoirs and/or monitoring of fluid flows in a reservoir [7] is often performed through an inversion process $[32,31,16]$. Such an inversion process requires multiple solutions of the EM forward model, which is often the most time consuming task of any inversion algorithm. The computational cost involved with solving the forward problem is dominated by the cost of solving the linear system arising from discretization of the forward model equations. 


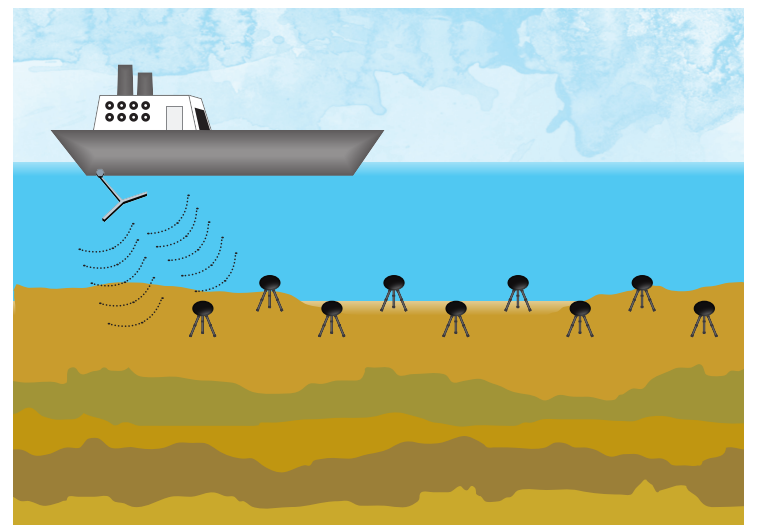

Fig. 1 Marine CSEM acquisition system employed for the characterization of the Earth's subsurface.

The forward problem associated with the simulation of three-dimensional (3D) marine CSEM measurements involves a large number of right-hand sides, due to the multiple source positions. Typically, the number of sources is in the order of tens to hundreds for land CSEM studies, while it reaches thousands in modern large-scale marine CSEM surveys [21]. Puzyrev et al. [25] evaluated modern direct solvers on large-scale geophysical simulations that previously were considered unachievable with these methods.

Direct matrix solvers for large geophysical 3D forward problems [33, 28, 30, 26, 24, 14, 19, 8, 35, 2,15] can be computationally expensive, both in terms of memory and CPU time. A way to overcome this limitation is by reducing the dimensionality of the problem, namely from 3D modeling to a 2.5D [23], 2D [4] and/or 1D [20] approximations that are only suitable for particular geometries.

Recently, Bakr et al. [5] proposed a mixed two domain decomposition Fourier finite element (DDFFE) method for the simulation of 3D marine CSEM measurements. The method combines $\mathrm{H}(\mathbf{c u r l})$ finite elements with Fourier basis functions. The zones of the computational domain where it is reasonable to represent geoelectric properties in 2D are discretized by combining 2D finite elements with a Fourier series $[23,3]$. The remaining part is discretized utilizing traditional $\mathrm{H}$ (curl) 3D finite element (FE) methods. The resulting discretization delivers highly accurate simulations of marine CSEM problems with arbitrary 3D geometries while it considerably reduces the computational complexity of full 3D FE simulations for typical marine CSEM problems. Ren et al. [27] present a domain decomposition approach for plane wave 3D EM modeling using Lagrange multipliers on the interfaces of the subdomains.
In here, we first extend the DDFFE method on [5] to the case of multiple (and not just two) subdomains. Thus, we can consider more realistic geometries that possibly incorporate bathymetry and/or multiple 3D regions. The method will be abbreviated MDDFFE. Additionally, in this work we introduce an "adaptive" dimensional decomposition of the model problem. Thus, when the background model is $1 \mathrm{D}$, we compute a $1.5 \mathrm{D}$ semi-analytical solution for the primary field, and we employ the MDDFFE method over the secondary field formulation. Similarly, when the background model is $2 \mathrm{D}$, we employ a $2.5 \mathrm{D}$ FE method to model the primary field. By doing so, we only need to factorize the $3 \mathrm{D}$ system matrix once (when employing direct solvers), and reuse the same factorization for all sources. This idea extends significantly traditional secondary field formulations where the primary field is computed over a homogeneous space [22], and provides further savings in the simulations. In particular, we avoid a 3D modeling of the singularities produced by the source, and we reduce the air layer effect, since these effects are mostly reproduced via the primary $1.5 \mathrm{D}$ (or $2.5 \mathrm{D})$ field.

The paper is organized as follows: Section 2 derives the formulation of the proposed MDDFFE method. Implementation details are described in Section 3. Numerical results included in Section 4 validate and analyze the convergence properties of the method, and also describe challenging simulations of marine CSEM measurements. Section 5 summarizes the main findings of this paper.

\section{Formulation of the method}

In this section, we first introduce time-harmonic Maxwell's equations. Then, we split the EM field into a primary and a secondary component. After that, we describe the corresponding $3 \mathrm{D}$ variational formulation of the secondary component. Subsequently, we split the 3D computational domain into subdomains exhibiting different dimensionality, and we introduce a special set of basis functions within each subdomain. Then, we derive the coupled variational formulation in terms of the subdomains.

\subsection{Time-harmonic Maxwell's equations}

EM phenomena is governed by Maxwell's equations. Assuming time variation of the form $e^{-i \omega t}$, and no free electric charges, Maxwell's equations in the frequency domain are given by:

$$
\nabla \times \mathbf{E}=i \omega \mu \mathbf{H}
$$




$$
\begin{aligned}
& \nabla \times \mathbf{H}=(\sigma-i \omega \epsilon) \mathbf{E}+\mathbf{J}, \\
& \nabla \cdot(\epsilon \mathbf{E})=0, \\
& \nabla \cdot(\mu \mathbf{H})=0,
\end{aligned}
$$

where $\omega$ is the angular frequency, $\mathbf{E}$ is the electric field, $\mathbf{H}$ is the magnetic field, $\mu$ is the magnetic permeability, $\epsilon$ is the permittivity, $\sigma$ is the electric conductivity, $\mathbf{J}$ is the source current distribution, and $i=\sqrt{-1}$. Notice that when $\omega \neq 0$, divergence equations (3) and (4) are redundant, and they do not need to be imposed, since they can be derived from the curl equations (1) and (2) by taking their divergence.

Pre-multiplying both sides of (1) by $\mu^{-1}$ (we assume that $\operatorname{det}(\mu) \neq 0$ and $\operatorname{det}(\sigma-i \omega \epsilon) \neq 0)$, applying the curl operator to that equation, and substituting (2) in the resulting equation, one eliminates $\mathbf{H}$ to obtain the socalled reduced wave equation in terms of the electric field

$\nabla \times\left(\mu^{-1} \nabla \times \mathbf{E}\right)-i \omega \tilde{\sigma} \mathbf{E}=i \omega \mathbf{J}$,

where $\tilde{\sigma}=\sigma-i \omega \epsilon$. Once $\mathbf{E}$ is known after solving (5), $\mathbf{H}$ can be obtained from Faraday's law, see Eq.(1). To ensure uniqueness of the solution, the perfect electrically conductive boundary condition is imposed:

$\left.(\mathbf{n} \times \mathbf{E})\right|_{\Gamma}=\mathbf{0}$,

where $\mathbf{n}$ is the unit normal vector outward to the boundary $\Gamma=\partial \Omega$ of a computational domain $\Omega \subseteq$ $R^{3}$. The truncation of the computational domain with boundary condition (6) does not cause significant reflections in the solution because the electric field amplitude decays exponentially fast in the diffusive region as we move away from the source. Thus, the above boundary condition can be imposed on a surface located sufficiently far from the source. We consider deep waters to ignore the effect of the air. For shallow waters, the effect of the air may become relevant [11], and one could need to add some type of absorbing boundary condition or absorbing layer, such as a perfectly matched layer [6].

\subsubsection{Secondary field formulation}

We decompose the conductivity distribution as the sum $\sigma=\sigma^{b}+\sigma^{a}$. Here, $\sigma^{b}$ indicates a background conductivity distribution and $\sigma^{a}$ denotes the "anomalous" conductivity. Correspondingly, $\mathbf{E}$ can be split into two parts $\mathbf{E}=\mathbf{E}^{b}+\mathbf{E}^{a}$. Here, $\mathbf{E}^{b}$ denotes the background field, that is, the field corresponding to conductivity $\sigma^{b}$, and $\mathbf{E}^{a}$ represents the part of the field caused by the existence of the anomaly $\sigma^{a}$. The same decomposition can be applied to $\mathbf{H}$.

From the definitions of $\mathbf{E}^{b}$ and $\mathbf{H}^{b}$, we obtain

$\nabla \times \mathbf{E}^{b}=i \omega \mu \mathbf{H}^{b}$,

$\nabla \times \mathbf{H}^{b}=\tilde{\sigma}^{b} \mathbf{E}^{b}+\mathbf{J}$ where $\tilde{\sigma}^{b}=\sigma^{b}-i \omega \epsilon$. By using the linearity of (1) and (2) and definition of $\mathbf{E}^{a}=\mathbf{E}-\mathbf{E}^{b}$ and $\mathbf{H}^{a}=\mathbf{H}-\mathbf{H}^{b}$, we obtain

$\nabla \times \mathbf{E}^{a}=i \omega \mu \mathbf{H}^{a}$,

$\nabla \times \mathbf{H}^{a}=\tilde{\sigma} \mathbf{E}^{a}+\sigma^{a} \mathbf{E}^{b}$.

Pre-multiplying both sides of (9) by $\mu^{-1}$, applying the curl operator to the resulting equation and substituting from (10), one eliminates $\mathbf{H}^{a}$ to obtain

$\nabla \times\left(\mu^{-1} \nabla \times \mathbf{E}^{a}\right)-i \omega \tilde{\sigma} \mathbf{E}^{a}=i \omega \sigma^{a} \mathbf{E}^{b}$.

\section{$2.2 \mathbf{E}^{a}$-variational formulation}

Pre-multiplying (11) by $\mathbf{F}^{*}$ ( $\mathbf{F}$ is an arbitrary test function and "*" denotes its conjugate transpose) and integrating over the domain $\Omega$, we obtain

$$
\begin{aligned}
& \int_{\Omega} \mathbf{F}^{*}\left(\nabla \times \mu^{-1} \nabla \times \mathbf{E}^{a}\right) d \Omega-i \omega \int_{\Omega} \mathbf{F}^{*} \tilde{\sigma} \mathbf{E}^{a} d \Omega \\
& =i \omega \int_{\Omega} \mathbf{F}^{*} \sigma^{a} \mathbf{E}^{b} d \Omega .
\end{aligned}
$$

The first integral on the left hand side of (12) involving the curl operator can be transformed using Green's theorem to

$$
\begin{aligned}
& \int_{\Omega} \mathbf{F}^{*}\left(\nabla \times \mu^{-1} \nabla \times \mathbf{E}^{a}\right) d \Omega \\
& =\int_{\Omega}(\nabla \times \mathbf{F})^{*}\left(\mu^{-1} \nabla \times \mathbf{E}^{a}\right) d \Omega \\
& \quad+\int_{\Gamma} \mathbf{F}^{*}\left(\mathbf{n} \times \mu^{-1} \nabla \times \mathbf{E}^{a}\right) d \Gamma .
\end{aligned}
$$

The outer boundary integral of (13) is transformed using vector identity $\mathbf{F}^{*}\left(\mathbf{n} \times \mu^{-1} \nabla \times \mathbf{G}\right)=-(\mathbf{n} \times$ $\left.\mathbf{F}^{*}\right)\left(\mu^{-1} \nabla \times \mathbf{G}\right)$ to

$$
\begin{aligned}
& \int_{\Gamma} \mathbf{F}^{*}\left(\mathbf{n} \times \mu^{-1} \nabla \times \mathbf{E}^{a}\right) d \Gamma \\
& \quad=-\int_{\Gamma}\left(\mathbf{n} \times \mathbf{F}^{*}\right)\left(\mu^{-1} \nabla \times \mathbf{E}^{a}\right) d \Gamma .
\end{aligned}
$$

We select arbitrary test functions $\mathbf{F}$ of the form

$$
\begin{aligned}
\mathbf{F} & \in H_{\Gamma}(\mathbf{c u r l} ; \Omega) \\
& =\left\{\mathbf{F} \in H(\mathbf{c u r l} ; \Omega):\left.(\mathbf{n} \times \mathbf{F})\right|_{\Gamma}=\mathbf{0}\right\} .
\end{aligned}
$$

Here, $H(\mathbf{c u r l} ; \Omega)=\left\{\mathbf{F} \in\left(\mathrm{L}^{2}(\Omega)\right)^{3}: \operatorname{curl} \mathbf{F} \in\left(\mathrm{L}^{2}(\Omega)\right)^{3}\right\}$ is the space of vector functions with $\mathbf{L}^{2}$-integrable curl. Due to the selection in (15), boundary integral (14) vanishes, and (12) becomes

$$
\begin{aligned}
& \int_{\Omega}(\nabla \times \mathbf{F})^{*} \mu^{-1}\left(\nabla \times \mathbf{E}^{a}\right) d \Omega-i \omega \int_{\Omega} \mathbf{F}^{*} \tilde{\sigma} \mathbf{E}^{a} d \Omega \\
& \quad=i \omega \int_{\Omega} \mathbf{F}^{*} \sigma^{a} \mathbf{E}^{b} d \Omega .
\end{aligned}
$$




\subsection{DDFFE formulation with multiple subdomains}

In this subsection, we first extend the DDFFE method [5] to multiple subdomains and then apply it to the secondary field formulation (16).

We assume that the computational domain $\Omega=$ $\Omega(x, y, z)$ can be expressed as a tensor product $\Omega=$ $\mathbb{R}(y) \times \Omega_{2 D}(x, z)$, and the cross-sectional area $\Omega_{2 D}$ can be split into $\Omega_{2 D, 1}$ and $\Omega_{2 D, 2}$. We further split $\Omega_{2 D, 2}$ as the union of $N_{s}$ non-connected subdomains $\Omega_{2 D, 2}^{i}, i=$ $1, \ldots, N_{s}$, that is, $\Omega_{2 D, 2}=\cup_{i=1}^{N_{s}} \Omega_{2 D, 2}^{i}$. Hence, the computational domain may be written as $\Omega=\Omega_{1} \cup \Omega_{2}$, where $\Omega_{1}=\mathbb{R}(y) \times \Omega_{2 D, 1}$ and $\Omega_{2}=\mathbb{R}(y) \times \Omega_{2 D, 2}$. Furthermore, the computational subdomain $\Omega_{2}=\cup_{i=1}^{N_{s}} \Omega_{2}^{i}$, where $\Omega_{2}^{i}=\mathbb{R}(y) \times \Omega_{2 D, 2}^{i}, \quad i=1, \ldots, N_{s}$. Figure 2 shows an example with four computational subdomains, $\Omega_{1}, \Omega_{2}^{1}, \Omega_{2}^{2}$ and $\Omega_{2}^{3}$. The subdomain $\Omega_{1}$ is se-

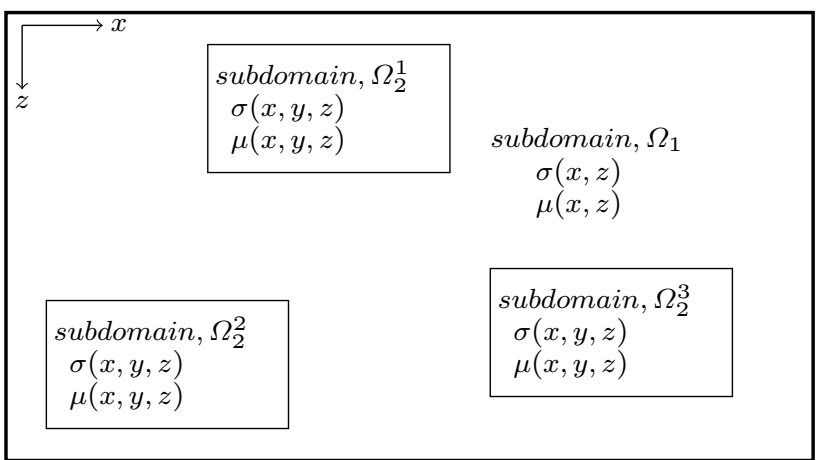

Fig. $2 x z$ cross section of an example with four computational subdomains and the material properties dependence within them.

lected with constant material properties $\mu$ and $\tilde{\sigma}$, with respect to the spatial variable $y$, i.e., $\mu=\mu_{2 D}(x, z)$ and $\tilde{\sigma}=\tilde{\sigma}_{2 D}(x, z)$. They are, however, allowed to vary in all three spatial directions in $\Omega_{2}$, that is $\mu=\mu(x, y, z)$ and $\tilde{\sigma}=\tilde{\sigma}(x, y, z)$.

The MDDFFE method expresses the solution of (16) as the sum of basis functions with support over $\Omega_{1}$ and a different set of basis functions with support over $\Omega_{2}^{i}, i=1,2, \ldots, N_{s}$ (for the case of two subdomains, see [5]). This results in the following hybrid representation:

$$
\begin{aligned}
\mathbf{E}^{a}(x, y, z) & =\sum_{m=-M}^{M} \sum_{n=1}^{N} E_{1, m n} \underbrace{\boldsymbol{\Phi}_{n}(x, z) e^{i r_{m} y}}_{\Omega_{1}} \\
& +\sum_{p=1}^{N_{s}} \sum_{k=1}^{K_{p}} E_{2, k p} \underbrace{\boldsymbol{\Psi}_{k p}(x, y, z)}_{\Omega_{2}^{p}} .
\end{aligned}
$$

Here, $\boldsymbol{\Psi}_{k p}(x, y, z)$ are basis functions with support over $\Omega_{2}^{p}$ corresponding to a discretization of $H\left(\mathbf{c u r l} ; \Omega_{2}^{p}\right)$, while mixed basis functions of the form $\boldsymbol{\Phi}_{n}(x, z) e^{i r_{m} y}$ are used for the discretization of $\Omega_{1}, E_{1, m n}$ and $E_{2, k p}$ are the unknowns (the so called degrees of freedom).

The $y$-independent part, $\boldsymbol{\Phi}_{n}(x, z)$, is a vectorvalued basis function with support over $\Omega_{2 D, 1}$. The $x-$ and $z$-components correspond to a discretization of $H\left(\mathbf{c u r l} ; \Omega_{2 D, 1}\right)$, while the $y$-component is associated with a discretization of $H^{1}\left(\Omega_{2 D, 1}\right)$. Here, $H^{1}(\Omega)=$ $\left\{F \in \mathrm{L}^{2}(\Omega): \nabla F \in\left(\mathrm{L}^{2}(\Omega)\right)^{3}\right\}$ is the space of scalar functions with $\mathrm{L}^{2}$-integrable gradient. Basically, in the subdomain $\Omega_{1}$ where materials are constant with respect to $y$, solution is expected to be smooth in that direction, and therefore, a high-order method is expected to converge exponentially fast in that subdomain. Therefore, one can employ Fourier basis functions, which are orthogonal, and provide a diagonal matrix while conserves the exponential convergence behavior.

Basis functions $e^{i r_{m} y}$ are the spectral Fourier representation of the field in the $y$-direction, with $r_{m}=\frac{2 \pi m}{T}$ being the $m$ - th Fourier mode. Selecting the number of Fourier modes according to the period $T=3 d$, where $d$ is the maximum distance between the source (in the case of equation (11) the source is the target body) and receivers, we obtained an acceptable accuracy in a pure $2.5 \mathrm{D}$ setting [23], and we follow the same prescription here.

Applying the curl operator to (17), we obtain

$$
\begin{aligned}
\nabla \times \mathbf{E}^{a} & =\sum_{m=-M}^{M} \sum_{n=1}^{N} E_{1, m n}\left(\nabla^{r_{m}} \times \boldsymbol{\Phi}_{n}(x, z)\right) e^{i r_{m} y} \\
& +\sum_{p=1}^{N_{s}} \sum_{k=1}^{K_{p}} E_{2, k p} \nabla \times \boldsymbol{\Psi}_{k p}(x, y, z),
\end{aligned}
$$

where $\nabla^{r_{m}}:=\left(\frac{\partial}{\partial x}, i r_{m}, \frac{\partial}{\partial z}\right)$.

We shall restrict ourselves to the Bubnov-Galerkin method. Selecting $\mathbf{F}=\boldsymbol{\Phi}_{j}(x, z) e^{i r_{s} y}, j=1, \ldots, N, s=$ $-M, \ldots, M$, as test functions, and substituting (17) and (18) into the variational formulation (16), we have

$$
\begin{aligned}
& \sum_{m=-M}^{M} \sum_{n=1}^{N} a_{s m}\left(\boldsymbol{\Phi}_{j}, \boldsymbol{\Phi}_{n}\right) E_{1, m n} \\
& +\sum_{p=1}^{N_{s}} \sum_{k=1}^{K_{p}} b_{s p}\left(\boldsymbol{\Phi}_{j}, \mathbf{\Psi}_{k p}\right) E_{2, k p} \\
& \quad=f_{1, s}\left(\mathbf{\Phi}_{j}\right), \quad j=1, \ldots, N, s=-M, \ldots, M,
\end{aligned}
$$

where

$$
\begin{aligned}
& a_{s m}\left(\mathbf{\Phi}_{j}, \boldsymbol{\Phi}_{n}\right) \\
& =\int_{\Omega}\left[\left(\nabla^{r_{s}} \times \boldsymbol{\Phi}_{j}\right)^{*} e^{-i r_{s} y}\left(\mu^{-1} \nabla^{r_{m}} \times \boldsymbol{\Phi}_{n}\right) e^{i r_{m} y}\right.
\end{aligned}
$$




$$
\left.-i \omega \boldsymbol{\Phi}_{j}^{*} e^{-i r_{s} y} \tilde{\sigma} \boldsymbol{\Phi}_{n} e^{i r_{m} y}\right] d \Omega,
$$

$$
\begin{array}{r}
b_{s p}\left(\boldsymbol{\Phi}_{j}, \mathbf{\Psi}_{k p}\right)=\int_{\Omega}\left[\left(\nabla^{r_{s}} \times \mathbf{\Phi}_{j}\right)^{*} e^{-i r_{s} y}\left(\mu^{-1} \nabla \times \mathbf{\Psi}_{k p}\right)\right. \\
\left.-i \omega \mathbf{\Phi}_{j}^{*} e^{-i r_{s} y} \tilde{\sigma} \mathbf{\Psi}_{k p}\right] d \Omega
\end{array}
$$

and

$f_{1, s}\left(\boldsymbol{\Phi}_{j}\right)=i \omega \int_{\Omega} \boldsymbol{\Phi}_{j}^{*} e^{-i r_{s} y} \sigma^{a} \mathbf{E}^{b} d \Omega$.

Selecting test functions of the form $\mathbf{F}=$ $\boldsymbol{\Psi}_{l q}(x, y, z), l=1, \ldots, K_{q}, q=1, \ldots, N_{s}$, system (19) becomes

$$
\begin{aligned}
& \sum_{m=-M}^{M} \sum_{n=1}^{N} c_{q m}\left(\boldsymbol{\Psi}_{l q}, \boldsymbol{\Phi}_{n}\right) E_{1, m n} \\
& \quad+\sum_{p=1}^{N_{s}} \sum_{k=1}^{K_{p}} d_{q p}\left(\boldsymbol{\Psi}_{l q}, \boldsymbol{\Psi}_{k p}\right) E_{2, k p} \\
& \quad=f_{2, q}\left(\mathbf{\Psi}_{l q}\right), \quad l=1, \ldots, K_{q}, q=1, \ldots, N_{s},
\end{aligned}
$$

where

$$
\begin{gathered}
c_{q m}\left(\mathbf{\Psi}_{l q}, \mathbf{\Phi}_{n}\right)=\int_{\Omega}\left[\left(\nabla \times \mathbf{\Psi}_{l q}\right)^{*}\left(\mu^{-1} \nabla^{r_{m}} \times \mathbf{\Phi}_{n}\right) e^{i r_{m} y}\right. \\
\left.-i \omega \mathbf{\Psi}_{l q}^{*} \tilde{\sigma} \boldsymbol{\Phi}_{n} e^{i r_{m} y}\right] d \Omega \\
d_{q p}\left(\boldsymbol{\Psi}_{l q}, \mathbf{\Psi}_{k p}\right)=\int_{\Omega}\left[\left(\nabla \times \mathbf{\Psi}_{l q}\right)^{*}\left(\mu^{-1} \nabla \times \mathbf{\Psi}_{k p}\right)\right. \\
\left.-i \omega \mathbf{\Psi}_{l q}^{*} \tilde{\sigma} \mathbf{\Psi}_{k p}\right] d \Omega .
\end{gathered}
$$

and

$f_{2, q}\left(\mathbf{\Psi}_{l q}\right)=i \omega \int_{\Omega} \boldsymbol{\Psi}_{l q}^{*} \sigma^{a} \mathbf{E}^{b} d \Omega$.

Utilizing the property that the material properties $\mu$ and $\tilde{\sigma}$ are $y$-independent on the support of $\boldsymbol{\Phi}_{j}(x, z), j=$ $1, \ldots, N,(20)$ can be expressed as:

$$
\begin{aligned}
a_{s m}\left(\boldsymbol{\Phi}_{j}, \boldsymbol{\Phi}_{n}\right) & =\int_{\Omega_{2 D, 1}}\left[\left(\nabla^{r_{s}} \times \boldsymbol{\Phi}_{j}\right)^{*}\left(\mu_{2 D}^{-1} \nabla^{r_{m}} \times \boldsymbol{\Phi}_{n}\right)\right. \\
& \left.-i \omega \boldsymbol{\Phi}_{j}^{*} \tilde{\sigma}_{2 D} \boldsymbol{\Phi}_{n}\right] \int_{\mathbb{R}} e^{-i\left(r_{s}-r_{m}\right) y} d y d \Omega_{2 D, 1} .
\end{aligned}
$$

By employing the orthogonality of the Fourier basis functions and the definition of the Kronecker's delta function, $\delta_{r_{s}, r_{m}}$, the above integrals become

$$
\begin{aligned}
a_{s m}\left(\boldsymbol{\Phi}_{j}, \boldsymbol{\Phi}_{n}\right)= & \int_{\Omega_{2 D, 1}}\left[\left(\nabla^{r_{s}} \times \boldsymbol{\Phi}_{j}\right)^{*}\left(\mu_{2 D}^{-1} \nabla^{r_{m}} \times \boldsymbol{\Phi}_{n}\right)\right. \\
& \left.-i \omega \boldsymbol{\Phi}_{j}^{*} \tilde{\sigma}_{2 D} \boldsymbol{\Phi}_{n}\right] 2 \pi \delta_{r_{s}, r_{m}} d \Omega_{2 D, 1} .
\end{aligned}
$$

From the properties of delta function, namely:

$$
\begin{aligned}
\delta_{r_{s}, r_{m}} & =\delta\left(r_{s}, r_{m}\right)=\delta\left(\frac{2 \pi s}{T}, \frac{2 \pi m}{T}\right)=\frac{T}{2 \pi} \delta(s, m) \\
& =\frac{T}{2 \pi}\left\{\begin{array}{l}
1 \quad s=m, \\
0 \text { otherwise }
\end{array}\right.
\end{aligned}
$$

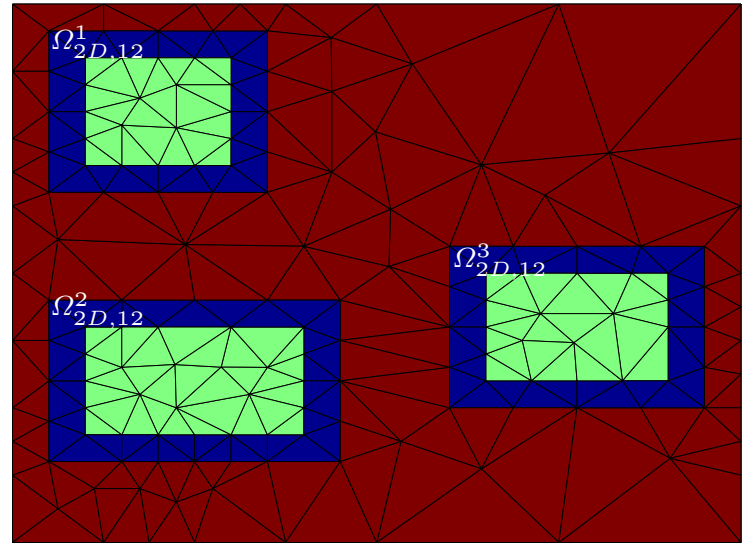

Fig. 3 Illustration of an example with three regions, $\Omega_{2 D, 12}^{i}, i=1,2,3$, where the coupling of the solution parts with support in $\Omega_{2 D, 1}$ and $\Omega_{2}^{i}, i=1,2,3$, respectively, take place.

we arrive at

$$
\begin{gathered}
\sum_{m=-M}^{M} \sum_{n=1}^{N} a_{s m}\left(\boldsymbol{\Phi}_{j}, \mathbf{\Phi}_{n}\right) E_{1, m n} \\
=T \sum_{n=1}^{N} a_{s s}\left(\boldsymbol{\Phi}_{j}, \mathbf{\Phi}_{n}\right) E_{1, s n},
\end{gathered}
$$

where

$$
\begin{aligned}
a_{s s}\left(\boldsymbol{\Phi}_{j}, \mathbf{\Phi}_{n}\right)= & \int_{\Omega_{2 D, 1}}\left[\left(\nabla^{r_{s}} \times \boldsymbol{\Phi}_{j}\right)^{*}\left(\mu_{2 D}^{-1} \nabla^{r_{s}} \times \boldsymbol{\Phi}_{n}\right)\right. \\
& \left.-i \omega \mathbf{\Phi}_{j}^{*} \tilde{\sigma}_{2 D} \boldsymbol{\Phi}_{n}\right] d \Omega_{2 D, 1} .
\end{aligned}
$$

The coefficients $b_{s p}\left(\boldsymbol{\Phi}_{j}, \boldsymbol{\Psi}_{k p}\right)$ and $c_{q m}\left(\boldsymbol{\Psi}_{l q}, \boldsymbol{\Phi}_{n}\right)$ are associated with coupling of the solution part with support in $\Omega_{1}$ to the solution part with support in $\Omega_{2}$. Since there is no overlap between $\Omega_{1}$ and $\Omega_{2}$, the coupling will occur in the immediate vicinity of their common boundary (only one layer of elements). In our implementation, the common support of all basis functions involved in the coupling, denoted as $\Omega_{2 D, 12}^{r}$, consists of those elements in $\Omega_{1}$ that are adjacent to $\Omega_{2}^{r}, r=1, \ldots, N_{s}$, see Fig. 3. The region of integration in (21) and (24) then reduces to $\mathbb{R}(y) \times \Omega_{2 D, 12}^{r}(x, z), r=1, \ldots, N_{s}$. Utilizing the $y$-independence of $\mu$ and $\tilde{\sigma}$ over $\Omega_{1}$, one may write:

$$
\begin{aligned}
& b_{s p}\left(\mathbf{\Phi}_{j}, \mathbf{\Psi}_{k p}\right) \\
& =\int_{\Omega_{2 D, 12}^{p}}\left[\left(\nabla^{r_{s}} \times \boldsymbol{\Phi}_{j}\right)^{*} \mu_{2 D}^{-1} \int_{\mathbb{R}} e^{-i r_{s} y}\left(\nabla \times \mathbf{\Psi}_{k p}\right) d y\right. \\
& \left.-i \omega \boldsymbol{\Phi}_{j}^{*} \tilde{\sigma}_{2 D} \int_{\mathbb{R}} e^{-i r_{s} y} \boldsymbol{\Psi}_{k p} d y\right] d \Omega_{2 D, 12}^{p},
\end{aligned}
$$

and

$$
\begin{aligned}
& c_{q m}\left(\boldsymbol{\Psi}_{l q}, \mathbf{\Phi}_{n}\right) \\
& =\int_{\Omega_{2 D, 12}^{q}}\left[\left(\int_{\mathbb{R}}\left(\nabla \times \mathbf{\Psi}_{l q}\right)^{*} e^{i r_{m} y} d y\right) \mu_{2 D}^{-1}\left(\nabla^{r_{m}} \times \mathbf{\Phi}_{n}\right)\right.
\end{aligned}
$$


$\left.-i \omega\left(\int_{\mathbb{R}} \boldsymbol{\Psi}_{l q}^{*} e^{i r_{m} y} d y\right) \tilde{\sigma}_{2 D} \boldsymbol{\Phi}_{n}\right] d \Omega_{2 D, 12}^{q}$.

By noticing that basis functions $\boldsymbol{\Psi}_{k q}, k=$ $1, \ldots, K_{q}, q=1, . ., N_{s}$ have support over $\Omega_{2}^{q}$ and the subdomains $\Omega_{2}^{i}, i=1, \ldots, N_{s}$ are non-connected, (25) can be expressed as:

$$
\begin{aligned}
d_{q q}\left(\mathbf{\Psi}_{l q}, \mathbf{\Psi}_{k q}\right)= & \int_{\Omega_{2}^{q}}\left[\left(\nabla \times \boldsymbol{\Psi}_{l q}\right)^{*}\left(\mu^{-1} \nabla \times \boldsymbol{\Psi}_{k q}\right)\right. \\
& \left.-i \omega \mathbf{\Psi}_{l q}^{*} \tilde{\sigma} \boldsymbol{\Psi}_{k q}\right] d \Omega_{2}^{q} .
\end{aligned}
$$

The right hand sides, (22) and (26), of (19) and (23) respectively, may be written as:

$f_{1, s}\left(\boldsymbol{\Phi}_{j}\right)=i \omega \int_{\Omega_{1}} \boldsymbol{\Phi}_{j}^{*} e^{-i r_{s} y} \sigma^{a} \mathbf{E}^{b} d \Omega_{1}$,

and

$f_{2, q}\left(\boldsymbol{\Psi}_{l q}\right)=i \omega \int_{\Omega_{2}^{q}} \boldsymbol{\Psi}_{l q}^{*} \sigma^{a} \mathbf{E}^{b} d \Omega_{2}^{q}$.

With the above expressions for the coefficients, $a_{s s}, b_{s p}, c_{q m}, d_{q q}, f_{1, s}$ and $f_{2, q}$, the resulting linear system becomes

$$
\begin{aligned}
& \sum_{n=1}^{N} a_{s s}\left(\boldsymbol{\Phi}_{j}, \boldsymbol{\Phi}_{n}\right) E_{1, s n}+\sum_{p=1}^{N_{s}} \sum_{k=1}^{K_{p}} b_{s p}\left(\boldsymbol{\Phi}_{j}, \boldsymbol{\Psi}_{k p}\right) E_{2, k p} \\
& \quad=f_{1, s}\left(\boldsymbol{\Phi}_{j}\right), j=1, \ldots, N, s=-M, \ldots, M \\
& \sum_{m=-M}^{M} \sum_{n=1}^{N} c_{q m}\left(\boldsymbol{\Psi}_{l q}, \boldsymbol{\Phi}_{n}\right) E_{1, m n}+\sum_{k=1}^{K_{q}} d_{q q}\left(\mathbf{\Psi}_{l q}, \mathbf{\Psi}_{k q}\right) E_{2, k q} \\
& \quad=f_{2, q}\left(\mathbf{\Psi}_{l q}\right), l=1, \ldots, K_{q}, q=1, \ldots, N_{s} .
\end{aligned}
$$

\section{Implementation}

In this section, we provide some implementation details about the proposed method.

\subsection{5D solution}

Analytical solutions for 1.5D Maxwell's equations are described in $[34,20]$ for the case when the Earth's subsurface can be modeled as a set of horizontally stratified layers with constant materials within each layer. By taking the Hankel transform $[1,17]$, we obtain the ordinary differential equation for the Hankel transform kernel. For a typical CSEM setup with a horizontal electric dipole (HED) source, the components of the electromagnetic fields $\mathbf{E}$ and $\mathbf{H}$ are given as integrals of the form

$\int_{0}^{\infty} f(\lambda) J_{i}(\lambda r) d \lambda$

The kernel function $f(\lambda)$ depends on the subsurface material properties, and $J_{i}(\lambda)$ is an $i$ - th order Bessel function of the first kind. Derivation of $\mathbf{E}$ and $\mathbf{H}$ on the form (35) can be found, e.g., in $[34,10]$.

In most 1.5D EM modelling codes, numerical evaluation of (35) is performed using a digital filter approach (see, e.g., $[1,17]$ ), which provides fast and accurate solutions. Integral (35) can also be approximated using quadrature methods (see, e.g., [9]), although they are less popular in geophysical EM applications.

\section{$3.22 .5 \mathrm{D}$ and $3 \mathrm{D}$ solutions}

For a 2D model, the solution method is based on a spatial discretization that combines a 1D Fourier transform with a 2D FE method [3]. We employ unstructured triangular grids [29] that permit efficient discretizations of geometrically complex domains.

Finally, for 3D models, we employ the MDDFFE method. To discretize the model domain $\Omega$, two types of meshes are constructed for subdomains $\Omega_{2 D}$ and $\Omega_{2}$. A $2 \mathrm{D}$ mesh is constructed by dividing $\Omega_{2 D}=\Omega_{2 D, 1} \cup$ $\Omega_{2 D, 2}$ into a number of triangular finite elements. The $3 \mathrm{D}$ mesh is obtained by extending a $2 \mathrm{D}$ triangular mesh over $\Omega_{2 D, 2}$ to a $3 \mathrm{D}$ prismatic mesh over $\Omega_{2}$.

In the current version of the MDDFFE code, implemented in MATLAB, we employ first order basis functions for all finite element spaces, and we assume that material properties are constant within a finite element. Thus, the coefficients in (33) and (34) can be evaluated analytically over each element. Summing up the integrals over all elements, the matrix form of (33) and (34) becomes:

$\left[\begin{array}{ll}\mathrm{A} & \mathrm{B} \\ \mathbf{C} & \mathrm{D}\end{array}\right]\left[\begin{array}{l}\mathbf{E}_{1} \\ \mathbf{E}_{2}\end{array}\right]=\left[\begin{array}{l}\mathbf{F}_{1} \\ \mathbf{F}_{2}\end{array}\right]$.

Here, D is block diagonal, with $N_{s}$ blocks, where each block $\mathbf{D}_{s}$ corresponds to a subdomain of $\Omega_{2}^{s}, s=$ $1, \ldots, N_{s}$ of size $K_{s} \times K_{s}$. The block diagonal matrix $\mathbf{D} \in \mathbb{C}^{K \times K}$ represents the discrete version of operator $\nabla \times \mu^{-1} \nabla \times-i \omega \tilde{\sigma}$ over $\Omega_{2}$, where $K=\sum_{s=1}^{N_{s}} K_{s}$. Hence, the sparsity of $\mathbf{D}$ corresponds to the sparsity of full $3 \mathrm{D}$ FE methods. The matrix $\mathbf{A} \in \mathbb{C}^{(2 M+1) N \times(2 M+1) N}$ is block diagonal, with $(2 M+1)$ blocks of size $N \times N$, where each block corresponds to a Fourier mode. The block diagonal structure of $\mathbf{A}$ is the result of the assumed $y$-independence of $\mu$ and $\tilde{\sigma}$ in $\Omega_{1}$ and the mutual orthogonality of the Fourier basis functions. Hence, $\mathbf{A}$ represents the discrete version of the operator $\nabla^{r_{s}} \times \mu_{2 D}^{-1} \nabla^{r_{s}} \times-i \omega \tilde{\sigma}_{2 D}$ over the domain $\Omega_{2 D, 1}$ for all involved Fourier modes. The sparsity of each diagonal block of $\mathbf{A}$ corresponds to the traditional sparsity of $2 \mathrm{D}$ FE methods. 
The rectangular matrix $\mathbf{B} \in \mathbb{C}^{(2 M+1) N \times K}$ is the discrete version of the operator $\nabla^{r_{s}} \times \mu_{2 D}^{-1} \nabla \times-i \omega \tilde{\sigma}_{2 D}$ over $\Omega_{2 D, 12}$ for all involved Fourier modes all subdomains, while the rectangular matrix $\mathbf{C} \in \mathbb{C}^{K \times(2 M+1) N}$ is the discrete version of the operator $\nabla \times \mu_{2 D}^{-1} \nabla^{r_{m}} \times-i \omega \tilde{\sigma}_{2 D}$ over $\Omega_{2 D, 12}$ for all involved Fourier modes and all subdomains. The matrices $\mathbf{B}$ and $\mathbf{C}$ are much more sparse than $\mathbf{A}$ and $\mathbf{D}$, since only a tiny fraction of the elements in $\Omega_{2 D, 1}$ and $\Omega_{2}$ are involved in the coupling along the common boundary of these subdomains. The column vectors $\mathbf{F}_{1} \in \mathbb{C}^{(2 M+1) N \times 1}$ and $\mathbf{F}_{2} \in \mathbb{C}^{K \times 1}$ are the discrete version of the right hand side of equation (22) over $\Omega_{1}$ and equation (26) over $\Omega_{2}$, respectively.

\section{Numerical results}

In this section, we first compare the performance and the relative error of the MDDFFE method when applied to solve the full field formulation given by equation (5) and the secondary field formulation (11). We also compare CPU times for full and secondary field formulations versus the relative error in percent. Finally, we perform more challenging numerical simulations over geometrically complex scenarios.

In all considered examples, the frequency excited by the horizontal electric dipole is equal to $0.25 \mathrm{~Hz}$. The coefficient functions, $\mu, \epsilon$ and $\sigma$, in Maxwell's equations, are assumed to be scalars. $\mu$ and $\epsilon$ have constant values, equal to $4 \pi \times 10^{-7} \mathrm{H} / \mathrm{m}$ and $8.85 \times 10^{-12} \mathrm{~F} / \mathrm{m}$, respectively, while $\sigma$ will be specified later for each example.

The horizontally stratified 1D geoelectric model considered here to compute the background field $\left(\mathbf{E}^{b}\right)$ for the first two models consists of an air layer with conductivity $10^{-8} \mathrm{~S} / \mathrm{m}$, a seawater layer with thickness $1.5 \mathrm{~km}$ and conductivity $3.33 \mathrm{~S} / \mathrm{m}$, a sea-bottom layer with thickness $1.1 \mathrm{~km}$ and conductivity $1.0 \mathrm{~S} / \mathrm{m}$, a layer with thickness $1.1 \mathrm{~km}$ and conductivity $0.5 \mathrm{~S} / \mathrm{m}$, a layer with thickness $500 \mathrm{~m}$ and conductivity $0.67 \mathrm{~S} / \mathrm{m}$, and a more resistive basement layer with conductivity $0.2 \mathrm{~S} / \mathrm{m}$, see Fig. 4 .

\subsection{Verification and convergence analysis}

The 3D model is derived from the 1D geoelectric one by including a 3D resistive target rectangular box (conductivity equals to $0.01 \mathrm{~S} / \mathrm{m}$ ) with finite size in all axes directions. The geoelectric model is illustrated in Fig. 4. The size of the resistive target (black color) is $3,000 \mathrm{~m}, 6,000 \mathrm{~m}$ and $100 \mathrm{~m}$ in the $x, y$ and $z$ directions, respectively. The transmitter is located $50 \mathrm{~m}$ above the sea-floor with $(x, y)$ coordinates equal to $(0,0)$. There are ten equally spaced receivers located on the sea-floor at horizontal distances varying from $1 \mathrm{~km}$ to $10 \mathrm{~km}$.

The MDDFFE method for the full field formulation (5) and the secondary field formulation (11) are compared to an integral equation (IE) method (see, e.g., [18]).

Figure 5 displays the amplitudes of $E_{x}$ and $E_{z}$ as functions of the horizontal distance between transmitter and receivers for the model problem described in Fig. 4. For the secondary field formulation, the amplitude of the electric field $\left|E_{\alpha}\right|$ is $\left|E_{\alpha}^{a}+E_{\alpha}^{b}\right|, \alpha=x, z$, which is the sum of the secondary field computed from equation (16) and the primary field given by the semianalytical $1.5 \mathrm{D}$ solution. Results obtained with the MDDFFE method with nine Fourier modes for the full field formulation (black dashed line with crosses) and for the secondary field formulation (black solid line) and IE method (red circles) agree very well.

Next, we show the relative error in percent, $\gamma_{\alpha}$, between the IE method and the MDDFFE method for the full field formulation and for the secondary field formulation, defined as,

$\gamma_{\alpha}=100 \frac{|| E_{\alpha}^{I E}\left(\mathbf{r}_{i}\right)|-| E_{\alpha}^{M D D F F E}\left(\mathbf{r}_{i}\right)||}{\left|E_{\alpha}^{I E}\left(\mathbf{r}_{i}\right)\right|}$,

where $\mathbf{r}_{i}$ denotes an arbitrary receiver, and $\alpha=x, z$. Figure 6 displays the relative error in percent of the amplitude of the electric field components $\gamma_{x}$ (left panel) and $\gamma_{z}$ (right panel) as functions of the number of unknowns for the model problem of Fig. 4. The MDDFFE method for the secondary field formulation delivers very accurate results with a significantly lower number of unknowns. About $25 \%$ of the unknowns is sufficient to provide a better accuracy when using the secondary field formulation. An error level below 10\% is acceptable and corresponds to what it is considered an accurate solution in the context of marine CSEM measurements, since the anomaly produced by the presence of an oil box is considerably larger (between $10 \%$ and $80 \%$, See Fig. 7).

Figure 8 displays the relative error in percent as a function of the number of Fourier modes for the model problem of Fig. 4. The MDDFFE method for the secondary field formulation delivers highly accurate results with a lower number of Fourier modes. About $50 \%$ of the number of Fourier modes is sufficient to provide a better accuracy when using the secondary field formulation in comparison to the full field formulation.

To summarize, from Figures 6 and 8, we conclude that the savings associated to the use of the secondary field formulation in terms of the number of $2 \mathrm{D}$ degrees of freedom and 1D Fourier modes are approximately factors of 4 and 2, respectively. Thus, the total savings 


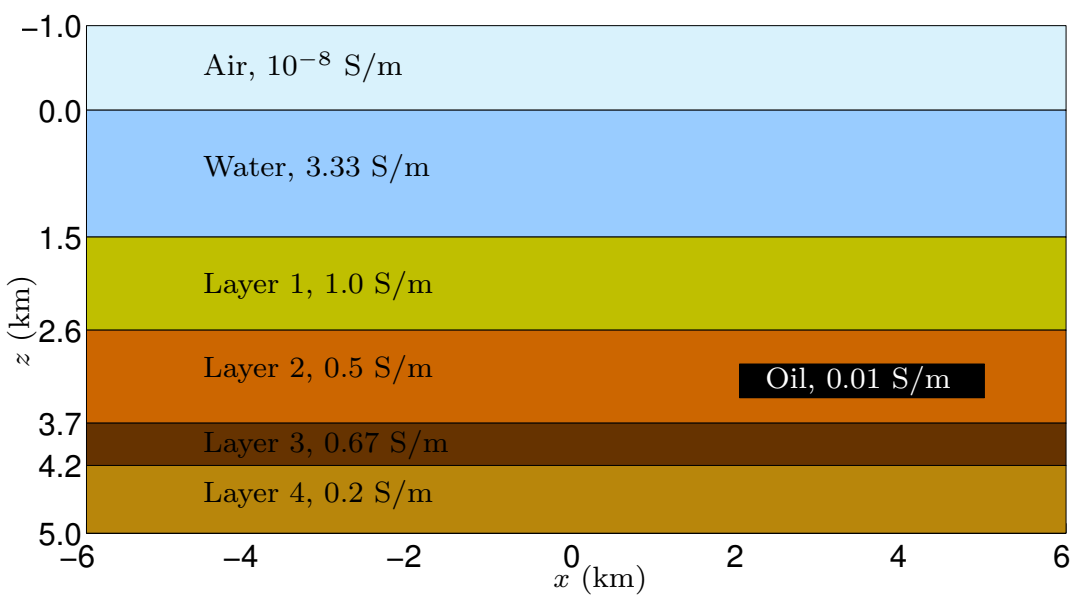

Fig. 4 Vertical cross section of the geoelectric model with a 3D target. Only the central portion of the model is shown.
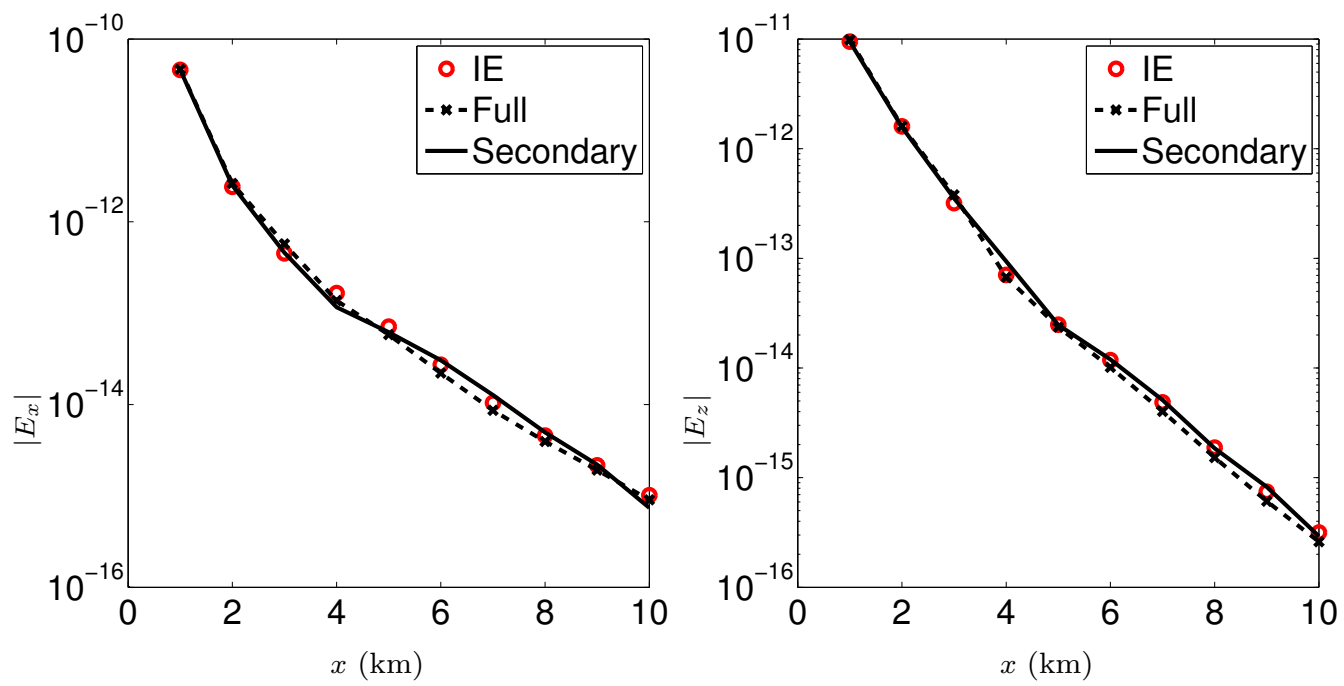

Fig. 5 Amplitudes of the electric field components $E_{x}$ (left panel) and $E_{z}$ (right panel) obtained with IE method (red circles) and the MDDFFE method with nine Fourier modes for the full field formulation (dashed line with crosses) and for the secondary field formulation (black solid line) as a function of the horizontal distance between transmitter and receivers.

amount to a factor of approximately 8 in the considered example. Therefore, about $13 \%$ of the unknowns is sufficient to provide a similar or even better accuracy when using the secondary field formulation.

Figure 9 displays CPU time in seconds used by the direct solver for the MDDFFE for the full and secondary field formulations vs. the relative error in percent, when applied to the model problem in Fig. 4. Tests were performed on a computer equipped with 16 GB RAM, and using only one core of the available 2.7 $\mathrm{GHz}$ dual-core processor. Results demonstrate that the secondary field formulation reduces the computational time by several orders of magnitude in order to achieve a comparable level of accuracy.

\subsection{Geophysical applications}

We now proceed to assess the performance of the new MDDFFE method on two 3D geoelectric models with bathymetry. In these two models, the MDDFFE method will only be compared to the 3D FE method, Since the assumptions underlying IE computations are violated for such geometrically complex geoelectric models.

\subsubsection{Geoelectric model with a 3D target and 3D bathymetry}

The geoelectric 3D model is illustrated in Fig. 10. This model has two 3D subdomains, namely a 3D target and 


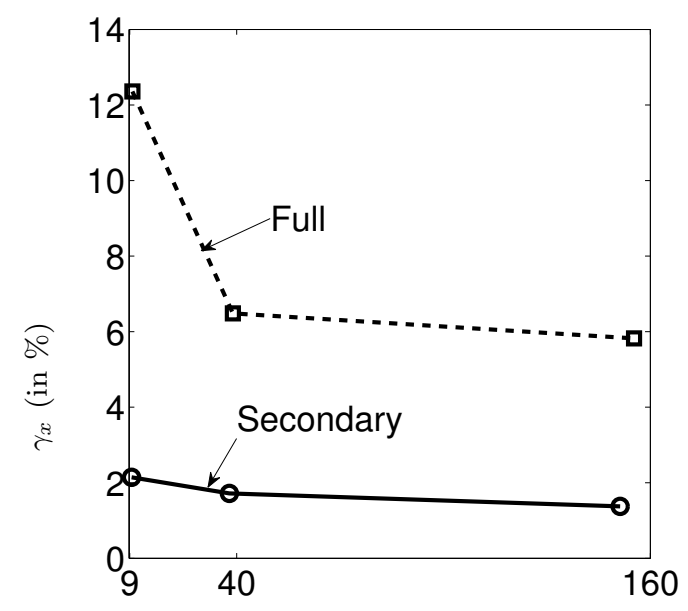

Number of unknowns $\times 10^{4}$

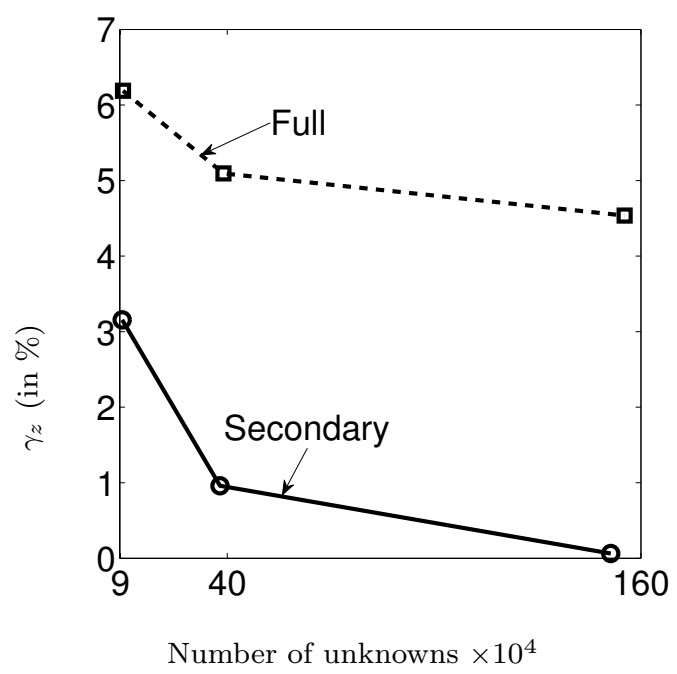

Fig. 6 Relative error (IE versus MDDFFE) in percent of the amplitude of the electric field components $\gamma_{x}$ (left panel) and $\gamma_{z}$ (right panel) obtained with the MDDFFE for the full field formulation (dashed line with squares) and the secondary field formulation (solid line with circles).
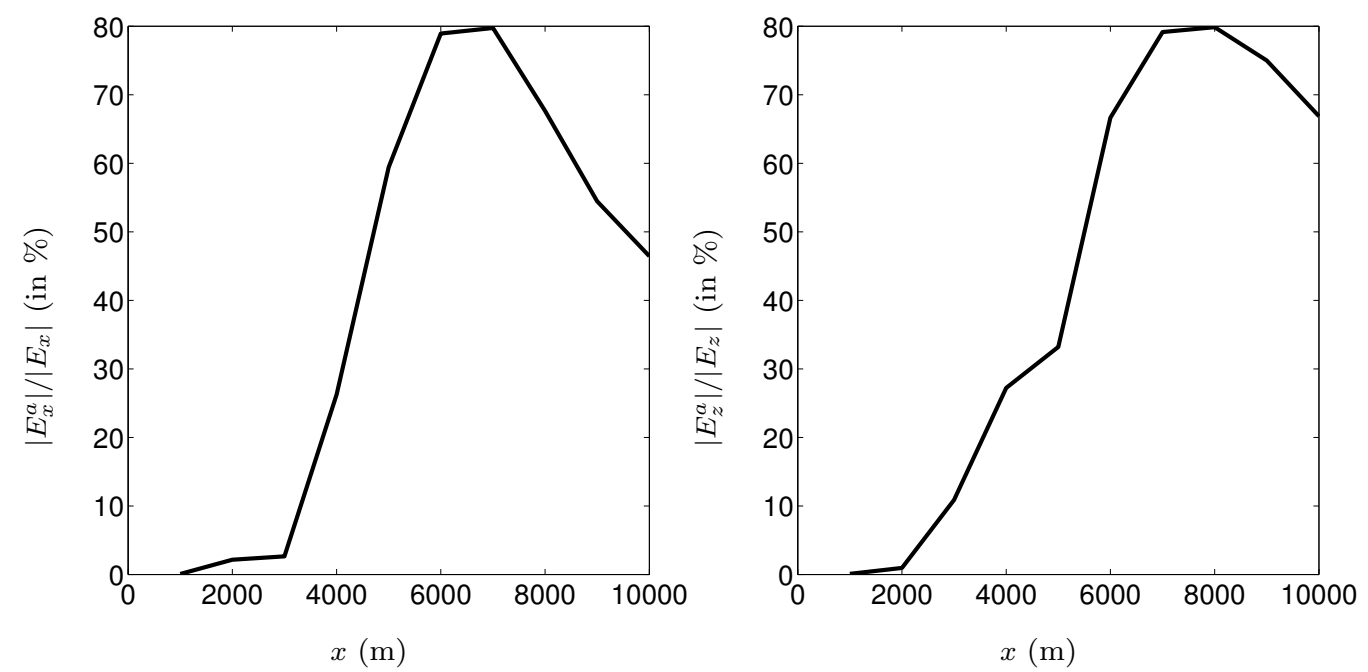

Fig. 7 The relative size of the anomaly (in percent) in terms of the amplitude of the electric field components due to the presence of an oil box.

that occupied by a 3D bathymetry. The $3 \mathrm{D}$ resistive target (conductivity equals to $0.01 \mathrm{~S} / \mathrm{m}$ ) has a finite size in all axes directions. The size of the resistive target (black color) is $3,000 \mathrm{~m}, 6,000 \mathrm{~m}$ and $100 \mathrm{~m}$ in the $x, y$ and $z$ directions, respectively. For a 3D bathymetry subdomain, we consider a trapezoidal-type hill model on the seafloor bathymetry, see Fig. 10. The 3D bathymetry subdomain is $100 \mathrm{~m}$ high, $200 \mathrm{~m}$ wide at the top and $1000 \mathrm{~m}$ wide at the bottom in $\mathrm{x}$-direction and $1000 \mathrm{~m}$ wide in y-direction.
The transmitter is located $100 \mathrm{~m}$ above the sea-floor with $(x, y)$ coordinates equal to $(-1500 \mathrm{~m}, 0 \mathrm{~m})$. The receivers are located at $y=0$ and along the bathymetry and on the sea-floor at horizontal distances varying from $-3 \mathrm{~km}$ to $3 \mathrm{~km}$.

Figure 11 displays the amplitudes of $E_{x}^{a}$ and $E_{z}^{a}$ at receivers positions for the model problem of Fig. 10. Results obtained with the MDDFFE method (black dashed line with crosses) and the 3D FE method (black solid line) show a good agreement. 

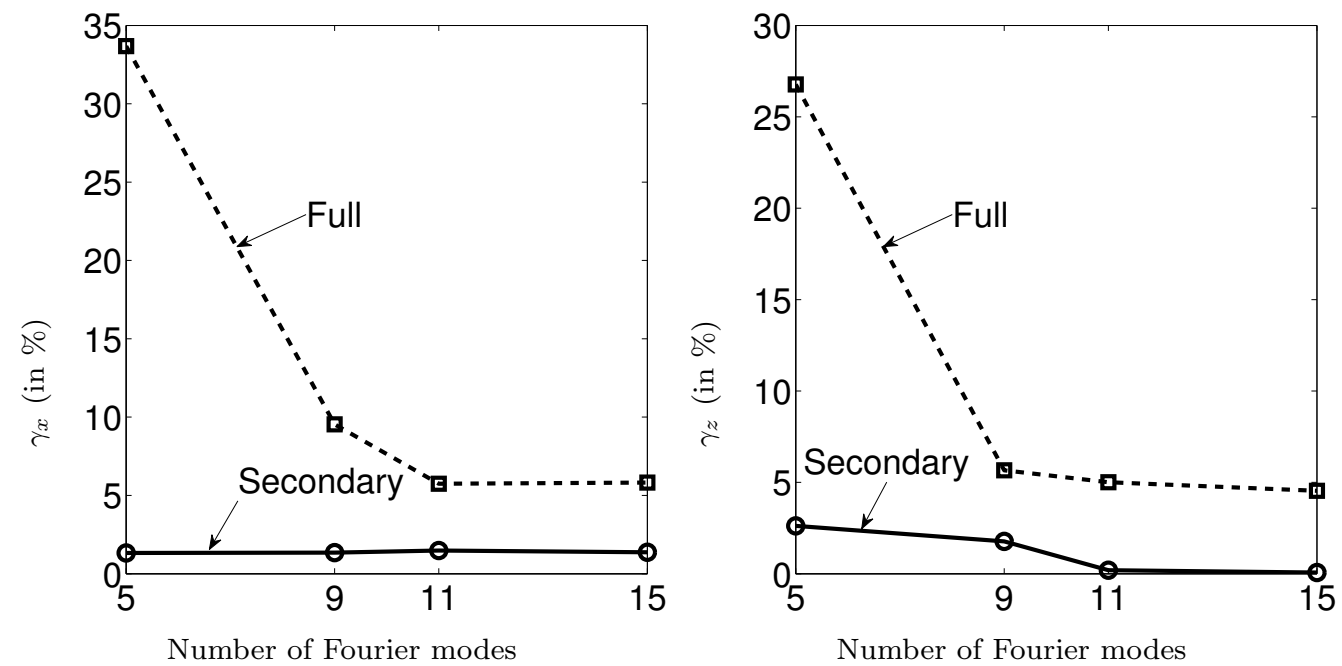

Fig. 8 Relative error (IE versus MDDFFE) in percent of the amplitude of the electric field components $\gamma_{x}$ (left panel) and $\gamma_{z}$ (right panel) obtained with the MDDFFE for the full field formulation (dashed line with squares) and the secondary field formulation (solid line with circles).

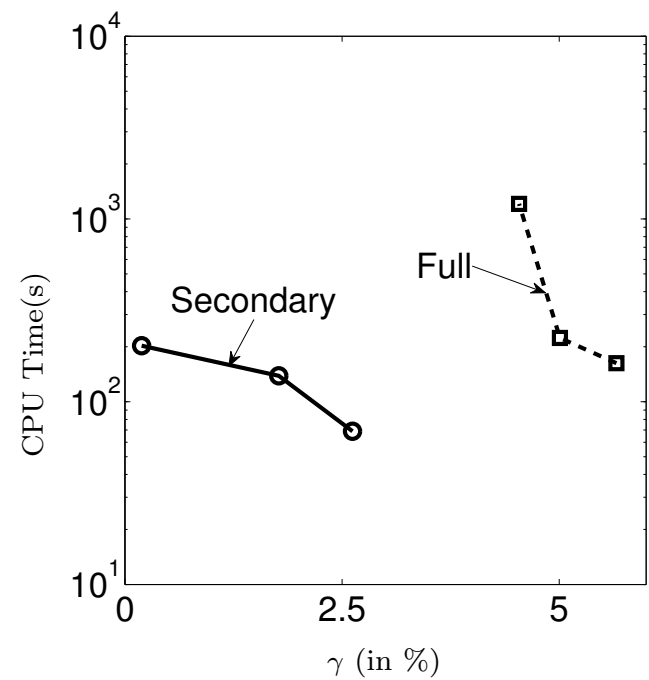

Fig. 9 CPU time used by the direct solver when employing the MDDFFE method for full and secondary field formulations vs. the relative error in percent when applied to the model problem of Fig. 4.

The number of unknowns used by the MDDFFE method and the 3D FE method is 375,201 and 677,095, respectively. The CPU times spent by the MDDFFE and the 3D FE methods are 1,134 and 3,311 seconds, respectively. For the MDDFFE method, the number of unknowns in the 2D subdomain, 3D bathymetry subdomain and 3D target subdomain is 315,053, 55,185 and 4,963 , respectively. In both methods, we employ the secondary field based formulation and we used the 1D model to compute the primary field.

\subsubsection{Complex 3D model with bathymetry}

We now assess the accuracy of the MDDFFE method on a complex 3D geoelectric model with a 2D bathymetry. We compute the primary electric field for the 2D background model using a 2.5D FE method. In this example, the 3D geoelectric model is illustrated in Fig. 12. The resistive target (black color, conductivity equal to $0.01 \mathrm{~S} / \mathrm{m}$ ), as well as the surrounding layers are described by a complex geometry. The transmitter is located $90 \mathrm{~m}$ above the sea-floor with $(x, y)$ coordinates 


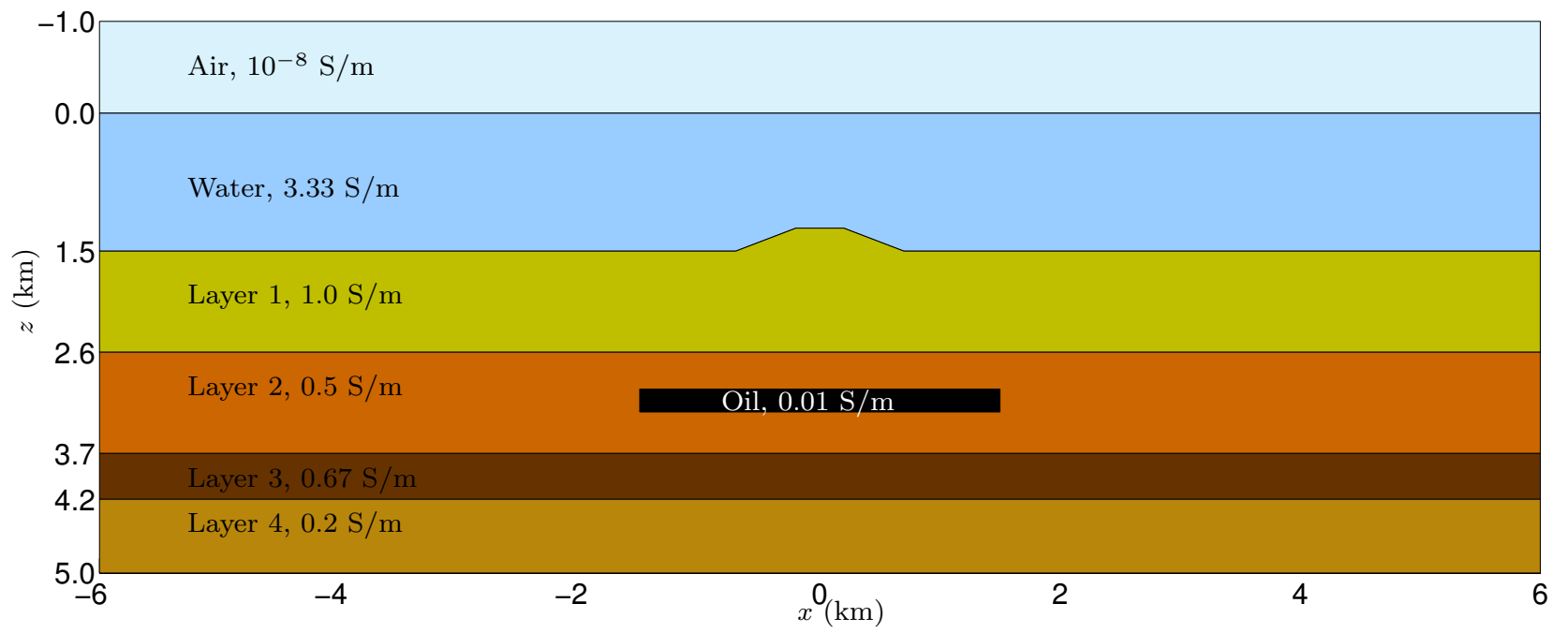

Fig. 10 Vertical cross section $(y=0)$ of the geoelectric model with a 3D target and 3D bathymetry. Only the central portion of the model is shown.
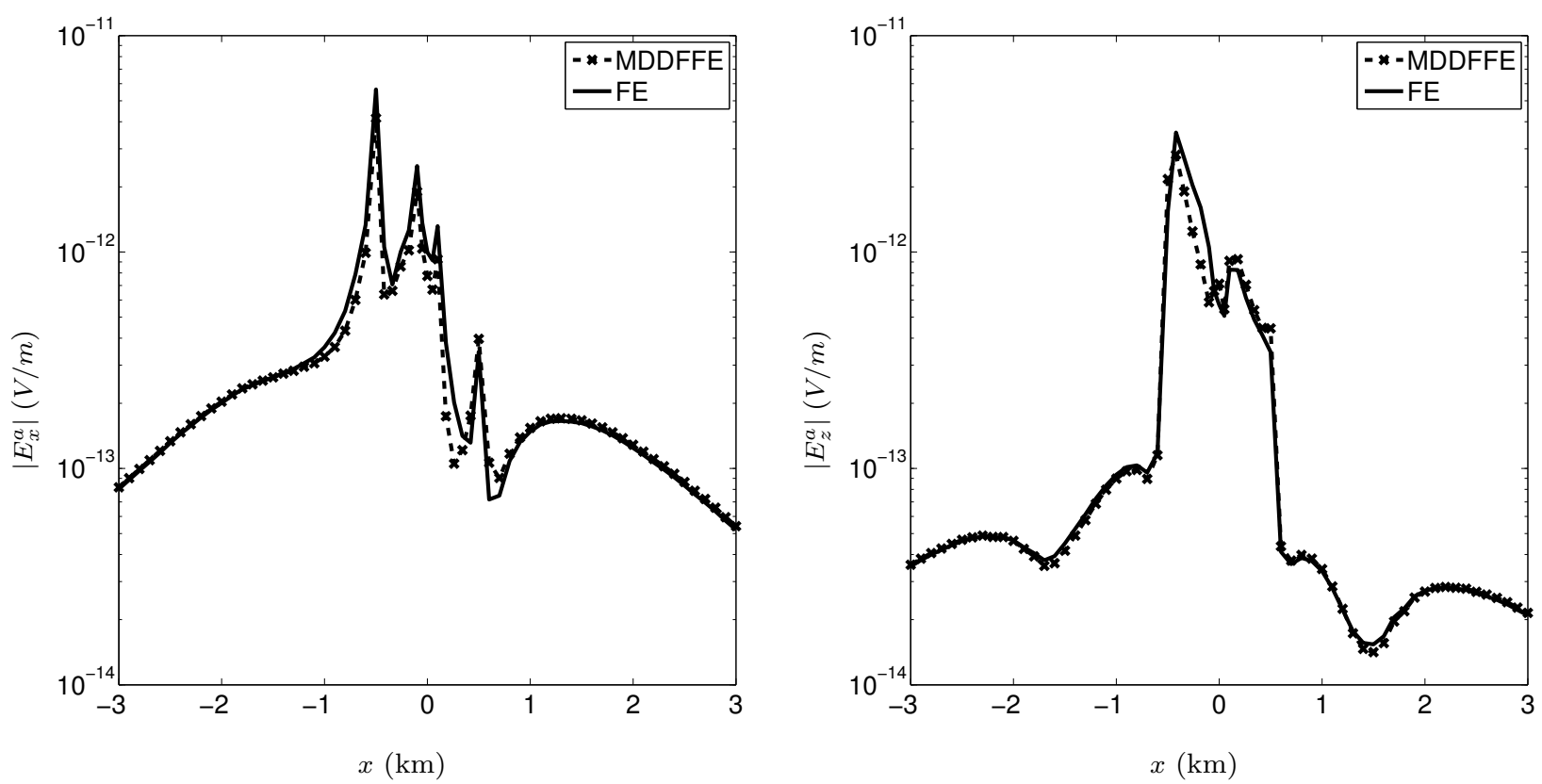

Fig. 11 Amplitudes of the electric field components $E_{x}^{a}$ (left panel) and $E_{z}^{a}$ (right panel) obtained with the MDDFFE method (black dashed line with crosses) and the 3D FE method (black solid line) at receivers positions.

equal to $(0,0)$. The receivers are located at $y=0$ and along the bathymetry at horizontal distances varying from $1 \mathrm{~km}$ to $23 \mathrm{~km}$.

Figure 13 displays the amplitudes of $E_{x}^{a}$ and $E_{z}^{a}$ as functions of the horizontal distance between transmitter and receivers for the model problem of Fig. 12. Results obtained with the MDDFFE method (black dashed line with crosses) and the 3D FE method (black solid line) perfectly match, again demonstrating the accuracy of the MDDFFE method.
The numbers of unknowns used by the MDDFFE method and the 3D FE method are 190,083 and 326,434 unknowns, respectively. The CPU times used by the MDDFFE and the 3D FE methods are 31 and 1,084 seconds, respectively. This large difference of CPU times occurs not only because of the savings in terms of the number of unknowns (a factor of 2), but mainly because of the sparser structure of the coefficient matrix of the MDDFFE method (since the amount of time needed to solve the problem using a direct solver scales approx- 


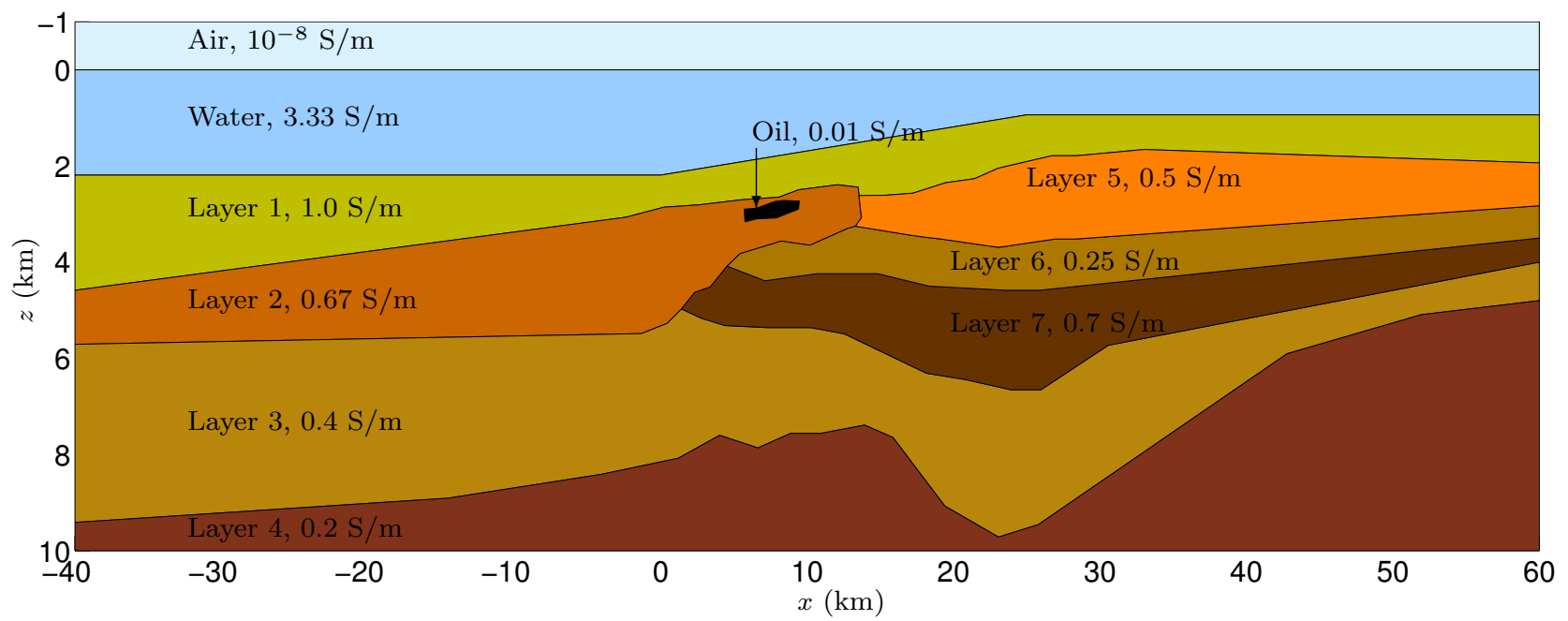

Fig. 12 Vertical cross section of a complex 3D geoelectric model. Only the central portion of the model in z-direction is shown.
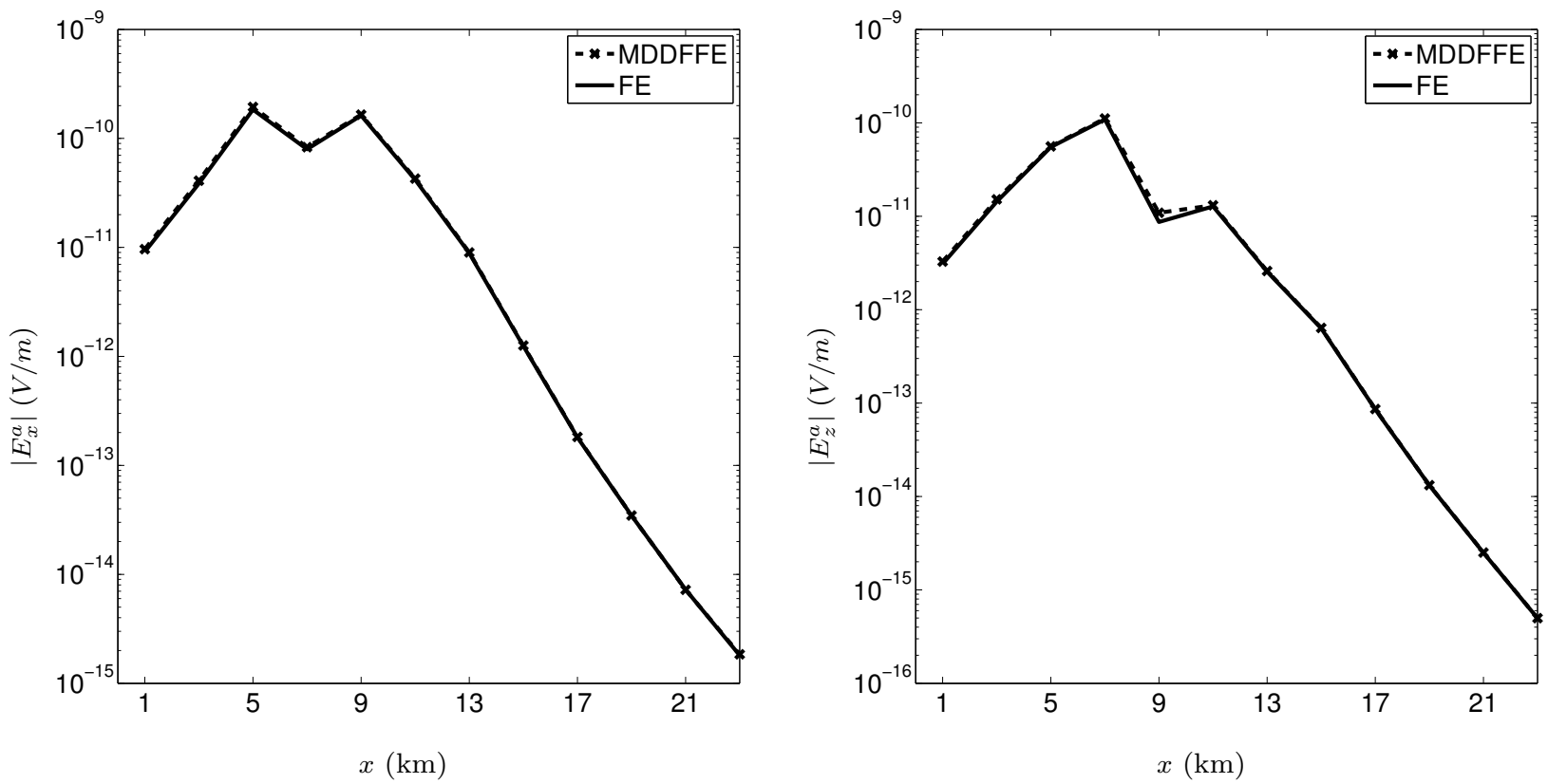

Fig. 13 Amplitudes of the electric field components $E_{x}^{a}$ (left panel) and $E_{z}^{a}$ (right panel) obtained with the MDDFFE method (black dashed line with crosses) and the 3D FE method (black solid line) as a function of the horizontal distance between transmitter and receivers.

imately as $N^{1.5}$ for the 2.5D FE methods, while $N^{2}$ for the 3D methods, and $N^{1.5}+N^{2}$ for the MDDFFE method, see the details of the computational complexity of DDFFE method in [5]). For the MDDFFE method, the number of unknowns in $\Omega_{1}$ and $\Omega_{2}$ are 185,070 and 5,013 unknowns, respectively. In both methods, we employ the secondary field based formulation and we used the 2.5D FE method to compute the primary field. The number of unknowns and the CPU times used by the
2.5D FE method are 146,433 and 4 seconds, respectively.

\section{Conclusions}

We have introduced a multi-domain decomposition Fourier finite element (MDDFFE) method for the simulation of 3D marine CSEM measurements. Starting from the full 3D model, a lower dimensional problem 
in the transverse direction has been considered. The resulting lower dimensional problem is solved using a $1.5 \mathrm{D}$ semi-analytical solution in the case of a $1 \mathrm{D}$ model, and a 2.5D FE method in the case of a 2D model, while higher dimensional effects are incorporated into the solution by solving for the secondary field selecting the previously computed lower dimensional solution as the primary field. We compared the performance and error of the secondary field formulation versus that obtained for the full field formulation. The accuracy of the MDDFFE method for both formulations was demonstrated to be superior to the one of traditional 3D FE methods through numerical comparison for typical 3D CSEM models. We showed numerically that the MDDFFE method for the secondary field formulation delivered very accurate results with a significantly lower number of unknowns when compared to the full field formulation. For the considered examples, about $13 \%$ of the unknowns is sufficient to provide a similar or even better accuracy when using the secondary field formulation. In some cases, the time savings were as large as a factor of 30 . We have applied the method to two challenging synthetic examples exhibiting bathymetry and multiple 3D subdomains.

\section{Acknowledgements}

This research is supported by the Basque Government through the BERC 2014-2017 program and by the Spanish Ministry of Economy and Competitiveness BCAM Severo Ochoa excellence accreditation SEV-2013-0323. David Pardo was partially funded by the RISE Horizon 2020 European Project GEAGAM (644602), the Project of the Spanish Ministry of Economy and Competitiveness with reference MTM201340824-P, and the Consolidated Research Group Grant IT649-13 on "Mathematical Modeling, Simulation, and Industrial Applications (M2SI)".

\section{References}

1. Anderson, W.L.: A hybrid fast Hankel transform algorithm for electromagnetic modeling. Geophysics 54(2), 263-266 (1989)

2. Ansari, S., Farquharson, C.G.: 3D finite-element forward modeling of electromagnetic data using vector and scalar potentials and unstructured grids. Geophysics $\mathbf{7 9}(4)$, E149-E165 (2014)

3. Bakr, S.A.: A 2.5D Finite element method for modeling of electromagnetic tensor Green's functions on a triangular mesh. Uni Research, Centre for Integrated Petroleum Research 1 (2012)

4. Bakr, S.A., Mannseth, T.: Feasibility of simplified integral equation modeling of low-frequency marine CSEM with a resistive target. Geophysics 74(5), F107-F117 (2009)

5. Bakr, S.A., Pardo, D., Mannseth, T.: Domain decomposition Fourier finite element method for the simulation of 3D marine CSEM measurements. J. Comput. Phys. 255, 456-470 (2013)

6. Berenger, J.: A perfectly matched layer for the absorption of electromagnetic waves. J. Comput. Phys. 114(2), 185200 (1994)

7. Berre, I., Lien, M., Mannseth, T.: Identification of threedimensional electric conductivity changes from timelapse electromagnetic observations. J. Comput. Phys. 230(10), 3915-3928 (2011)

8. Cai, H., Xiong, B., Han, M., Zhdanov, M.: 3D controlledsource electromagnetic modeling in anisotropic medium using edge-based finite element method. Computers \& Geosciences 73, 164-176 (2014)

9. Chave, A.D.: Numerical integration of related Hankel transforms by quadrature and continued fraction expansion. Geophysics 48(12), 1671-1686 (1983)

10. Chave, A.D.: On the electromagnetic fields produced by marine frequency domain controlled sources. Geophys. J. Int. 179(3), 1429-1457 (2009)

11. Constable, S.: Ten years of marine CSEM for hydrocarbon exploration. Geophysics 75(5), 75A67-75A81 (2010)

12. Constable, S., Srnka, L.J.: An introduction to marine controlled-source electromagnetic methods for hydrocarbon exploration. Geophysics 72(2), WA3-WA12 (2007)

13. Eidesmo, T., Ellingsrud, S., MacGregor, L.M., Constable, S., Sinha, M.C., Johansen, S.E., Kong, F.N., Westerdahl, H.: Sea bed logging (SBL), a new method for remote and direct identification of hydrocarbon filled layers in deepwater areas. First break 20(3), 144-152 (2002)

14. Grayver, A.V., Burg, M.: Robust and scalable 3-D geoelectromagnetic modelling approach using the finite element method. Geophys. J. Int. 198, 110-125 (2014)

15. Grayver, A.V., Kolev, T.V.: Large-scale 3D geoelectromagnetic modeling using parallel adaptive higher-order finite element method. Geophysics 80(6), E277-E291 (2015)

16. Grayver, A.V., Streich, R., Ritter, O.: 3D inversion and resolution analysis of land-based CSEM data from the Ketzin CO2 storage formation. Geophysics 79(2), E101E114 (2014)

17. Guptasarma, D., Singh, B.: New digital linear filters for Hankel $\mathrm{J}(0)$ and $\mathrm{J}(1)$ transforms. Geophysical Prospecting 45, 745-762 (1997)

18. Hursán, G., Zhdanov, M.: Contraction integral method in three-dimensional electromagnetic modeling. Radio Science 37, 1089 (2002)

19. Koldan, J., Puzyrev, V., de la Puente, J., Houzeaux, G., Cela, J.M.: Algebraic multigrid preconditioning within parallel finite-element solvers for 3-D electromagnetic modelling problems in geophysics. Geophys. J. Int. 197, 1442-1458 (2014)

20. Løseth, L.O., Ursin, B.: Electromagnetic fields in planarly layered anisotropic media. Geophys. J. Int. 170, 44-80 (2007)

21. Newman, G.A.: A review of high-performance computational strategies for modeling and imaging of electromagnetic induction data. Surv. Geophys. 35(1), 85-100 (2014)

22. Newman, G.A., Alumbaugh, D.L.: Frequency-domain modelling of airborne electromagnetic responses using staggered finite differences. Geophysical Prospecting 43, 1021-1042 (1995) 
23. Pardo, D., Nam, M.J., Torres-Verdin, C., Hoversten, M.G., Garay, I.: Simulation of marine controlled source electromagnetic measurements using a parallel Fourier hp-finite element method. Comput. Geosci. 15, 53-67 (2011)

24. Puzyrev, V., Koldan, J., de la Puente, J., Houzeaux, G., Vázquez, M., Cela, J.M.: A parallel finite-element method for three-dimensional controlled-source electromagnetic forward modelling. Geophys. J. Int. 193(2), 678-693 (2013)

25. Puzyrev, V., Koric, S., Wilkin, S.: Evaluation of parallel direct sparse linear solvers in electromagnetic geophysical problems. Computers \& Geosciences 89, 79-87 (2016)

26. Ren, Z., Kalscheuer, T., Greenhalgh, S., Maurer, H.: A goal-oriented adaptive finite-element approach for plane wave $3-\mathrm{D}$ electromagnetic modelling. Geophys. J. int. 194, 700-718 (2013)

27. Ren, Z., Kalscheuer, T., Greenhalgh, S., Maurer, H.: A finite-element-based domain-decomposition approach for plane wave 3D electromagnetic modeling. Geophysics 79(6), E255-E268 (2014)

28. Schwarzbach, C., Borner, R.U., Spitzer, K.: Threedimensional adaptive higher order finite element simulation for geo-electromagnetics- a marine CSEM example. Geophys. J. Int. 187(1), 63-74 (2011)

29. Shewchuk, J.R.: Triangle: engineering a 2D quality mesh generator and Delaunay triangulator. in: Applied Computational Geometry: Towards Geometric Engineering 1148, 203-222 (1996)

30. da Silva, N.V., Morgan, J.V., MacGregor, L., Warner, M.: A finite element multifrontal method for 3D CSEM modeling in the frequency domain. Geophysics $\mathbf{7 7}(2)$, E101-E115 (2012)

31. Tveit, S., Bakr, S.A., Lien, M., Mannseth, T.: Ensemblebased Bayesian inversion of CSEM data for subsurface structure identification. Geophys. J. Int. 201, 1849-1867 (2015)

32. Tveit, S., Bakr, S.A., Lien, M., Mannseth, T.: Identification of subsurface structures using electromagnetic data and shape priors. J. Comput. Phys. 284, 505-527 (2015)

33. Um, E.S., Harris, J.M., Alumbaugh, D.L.: 3D timedomain simulation of electromagnetic diffusion phenomena: A finite-element electric-field approach. Geophysics 75(4), F115-F126 (2010)

34. Ward, S.H., Hohmann, G.W.: Electromagnetic Theory for Geophysical Applications. In Electromagn. Methods Appl. Geophys. Volume 1, Theory., Society of Exploration Geophysicists (1988)

35. Weiss, C.J.: Project APhiD: A Lorenz-gauged A $-\Phi$ decomposition for parallelized computation of ultrabroadband electromagnetic induction in a fully heterogeneous Earth. Computers \& Geosciences 58, 40-52 (2013) 